\title{
Estructura de costos de una PYME artesanal dedicada al área de bordado en el Departamento de Masaya
}

\author{
Br. Alicia Alejandra Novoa Aragón, Br. Walter José Useda Rodríguez \\ Tutor: Msc. Nancy Elizabeth Merlo Guerrero \\ Facultad de Ciencias Económicas, Departamento de Contaduría Pública Y Finanzas
}

\begin{abstract}
RESUMEN
En el presente ensayo se pretende abordar uno de los aspectos más trascendentales que interviene en el crecimiento y desarrollo de la economía nicaragüense, referenciado a la creciente participación económica y socio-cultural de parte de las PYME's artesanales de Masaya, localidad donde se concentran la mayor parte de artesanos. Estas, aportan considerablemente a la economía nacional pero son propensos a su extinción misma debido a la poca experiencia en cuanto a la estructuración de costos que integre los elementos de un determinado producto. Por lo cual, la temática expuesta se delimita a la estructuración de costos de un PYME artesanal dedicada al área de bordado en el Departamento de Masaya, realizando así, un proceso de investigación documental con nivel descriptivo, y, practicando a la vez un estudio de campo. Las directrices para la elaboración del ensayo se contemplan en la identificación y fundamento de la relevancia que conlleva la aplicación de una eficiente estructura de costos en una PYME artesanal, independientemente del tipo de manufactura que se realice.
\end{abstract}

Palabra Clave: PYME artesanal, Proceso productivo, Debilidades, Oportunidades, Desarrollo, Costos SUMMARY

In this essay seeks to address one of the most significant aspects involved in the growth and development of the Nicaraguan economy, referenced to the growing economic and socio-cultural participation from craft SME's of Masaya, city where most craftsmen live. These significantly contribute to the national economy but are prone to extinction due to very little experience in terms of cost structure that integrates the elements of a given product. Therefore, the theme is delimited exposed to cost structuring a craft dedicated to SMEs embroidery area in Masaya City, thereby performing a process of documental research with descriptive level, and, while practicing a field study. The guidelines for the preparation of the essay referred to the identification and foundament of relevance that involves the application of an efficient cost structure in a craft SMEs, regardless of the type of manufacturing being done.

Key word:SMEs artisanal production process, Weaknesses, Opportunities, Development, Costs

\section{INTRODUCCIÓN}

La actividad artesanal es un sector que se caracteriza por aportar considerablemente a la economía nicaragüense, concentrándose mayormente en el departamento de Masaya. Dicha actividad se distingue por el estilo típico y tradicional que se trasmite de generación en generación. 
Es significativo en relación a la cantidad de artesanos y al aporte económico a la economía nacional, pero existen factores que afectan la productividad y competitividad tales como: falta de financiamiento, organización, alto precio de la materia prima e insumos, poco conocimiento sobre técnicas de ventas y de mercado, bajos niveles de tecnificación en sus procesos, falta de densidad institucional tanto en los gremios de artesanos como en el resto de actores locales. La mayoría de las PYME's de este sector son de carácter familiar, transmitiendo a las generaciones subsecuentes una cultura de desarrollo basado en una labor tradicional.

Para la elaboración del presente ensayo se ha tomado como referencia una PYME artesanal dedicada al área de bordado en el Departamento de Masaya, cuyo enfoque está referido a fundamentar cuán relevante es la estructuración de costos para una determinada actividad productiva en el ramo artesanal.

\section{DESARROLLO DEL TEMA}

\section{Aspectos generales de la actividad artesanal del Departamento de Masaya}

A pesar de ser el más pequeño de los departamentos del país, Masaya es uno de los más poblados y plagados de diversos atractivos. Es un destino cultural importante debido a las latentes expresiones folclóricas populares que se originaron y hasta hoy se manifiestan en este pueblo.

Dentro de los principales materiales utilizados en la producción de artesanía nicaragüense encontramos: barro, marmolina, piedra, madera, pieles y cueros, cabuya, mimbre, bambú, tule, pita, textiles, frutos secos, hojas de elote, conchas de mar, coral, carey, jícaros, animales disecados, azúcar, metales y materiales de reciclaje, entre otros. Estos se han constituido en elementos fundamentales para el artesano nicaragüense, trabajándolos por muchos años y creando de ellos impresionantes artesanías, distintivas en cuanto a la creatividad y representación tradicional.

Con el Gobierno del Compañero Presidente Daniel Ortega Saavedra, la producción de artesanías en Nicaragua se ha diversificado y mejorado en cuanto a su calidad; permitiendo un reconocimiento a nivel centroamericano como la poseedora de la mayor variedad de artesanías con diversos productos de belleza, destacándose además con un ventajoso prestigio internacional.

\section{Generalidades de la PYME en Nicaragua}

Una de las definiciones claves dentro del ámbito de las micro, pequeñas y medianas empresas (MIPYME) es el tamaño de las empresas. Tradicionalmente, la clasificación de éstas se hace de acuerdo con el número de empleados. Aunque hay desventajas en este método, se utiliza una misma variable para la definición.

Microempresa: 1-5 empleados (FTE ${ }^{1}$ - Pequeña empresa: 6-20 empleados (FTE) - Mediana empresa: 21-50 empleados (FTE) - Gran empresa: 51 o más empleados (FTE)

\footnotetext{
${ }^{1}$ FTE significa full time equivalent el equivalente de un trabajador que labora tiempo completo
} 
Vale notar que la clasificación de empresas no es uniforme en el mundo. En general, se podría decir que entre más grande es una economía, más altos son los límites para ser una mediana o gran empresa.

En cuanto a la ubicación, las empresas en el sector PYME de Nicaragua se conglomeran mayormente en la zona del Pacífico del país.

\section{La PYME artesanal como factor fundamental en la economía nicaraguiense}

Cabe recalcar que, la producción de artesanías corresponde a las micro y pequeñas empresas del sector manufacturero, y, en conformidad a datos proporcionados por el $\mathrm{MEFCCA}^{2}$, se contabiliza un total de 18,457 empresas, que constituyen el 17.33\% del total de 106,500 MIPYMES existentes en el país. Además, el $55 \%$ de la artesanía del país provienen del departamento de Masaya; y el $45 \%$, de otros municipios del país.

De acuerdo a cifras oficiales emitidas por el Banco Central de Nicaragua, el aporte de las Micro, Pequeñas y Medianas empresas representa hasta un 40\%, aun existiendo capacitación técnica poco suficiente de parte de los propietarios en cuanto a la mejora y ampliación de sus negocios.

Las micro y pequeñas empresas son entornos que requieren mejorar su competitividad, y en referencia a ello, el especialista Mario Amador presidente de $\mathrm{CADIN}^{3}$; infiere la necesidad de potenciar las capacidades gerenciales para que estos sectores puedan contar con todas las herramientas de los conocimientos técnicos para la administración de sus negocios.

Un factor influyente es la intervención estatal, pues ha creado un mecanismo jurídico que ayuda a mejorar el clima de los negocios; en relación a esto, se hace referencia a la ley MIPYME $645^{4}$, la cual consagra las normativas, deberes y garantías para este sector.

\section{Dificultades y debilidades de las PYME's artesanales}

Generalmente las dificultades que inciden negativamente en la productividad de la PYME's son la obtención de crédito y el factor decisivo de operar en una economía informal. En cuanto al área de bordados encontramos otras dificultades latentes referidas a la mano de obra, estilo actual y la competencia existente no solo entre los artesanos sino entre las grandes empresas textiles de zonas francas.

La mano de obra es un recurso decadente que requiere de una capacitación técnica sistemática en el ramo artesanal, y en la actualidad, el arte del bordado es una especialidad que se está perdiendo debido al poco interés de la población de transmitir una tradición a sus generaciones sucesoras.

Por otro lado, el estilo del bordado artesanal y el diseño tradicional de sus prendas se constituye en un factor negativo, pues en un contexto actual existe una lucha entre la moda y la creación típica del

\footnotetext{
${ }^{2}$ Ministerio de la Economía Familiar, Comunitaria, Cooperativa y Asociativa

${ }^{3}$ Cámara de Industrias de Nicaragua

${ }^{4}$ Ley de Promoción, Fomento y Desarrollo de la Micro, Pequeña y Mediana Empresa (LEY MIPYME)
} 
vestuario textil nacional, donde un considerable número poblacional se inclina a las tendencias actuales con estilos de un mercado externo.

La competencia es considerable entre los mismos artesanos del sector de bordado; esto en referencia a la calidad del producto y a la capacidad empírica del propietario para llegar a sus clientes. Generalmente, en este ambiente las prendas son imitadas entre los mismos productores diferenciándolos a través de los clientes, los cuales se inclinan ya sea por la mejor oferta o por una mejor calidad.

Por otro lado, las zonas francas se instituyen en un pilar poco benéfico para la actividad del vestuario textil artesanal en vista de que son sectores distinguidos por su considerable volumen de producción y la oferta a un mercado tanto nacional como internacional.

En el mismo orden de ideas, otra de las debilidades que afecta la productividad del bordado artesanal es la falta de un sistema contable. Cabe señalar que, como parte de la planificación financiera, un adecuado sistema contable otorga credibilidad a cualquier actividad operativa, ofreciendo un conocimiento inmediato en cuanto a los ingresos y egresos generados en un determinado periodo, $\mathrm{y}$, metodológicamente permite un correcto manejo del inventario.

He aquí emerge otra de las debilidades que afecta a tal sector, la falta de estructuración de costos, ya que el manejo contable de estos, además de ser realizado por los propietarios es ejecutado de forma empírica, afectando de cierto modo la realización de un completo informe financiero que pueda ser ejecutado por usuarios tanto internos como externos. En consecuencia, los juicios de valor en cuanto a los márgenes de ganancia tienden a ser estimados de forma incorrecta, a veces aproximándose al valor real y otras no logran determinar los costos incurridos con exactitud aplicados al proceso de manufactura.

\section{Importancia de la estructura de costos en una PYME artesanal}

En principio, la Estructura de costos es caracterizado por ser un proceso orientado a organizar de manera práctica la gestión de costos, basado en las prioridades estratégicas y operativas de la organización.

Las disposiciones que anteceden aparentemente dan la pauta de que tal proceso puede ser aplicado únicamente a empresas de gran magnitud y con vasta experiencia. Sin embargo, se debe entender que cualquier proceso productivo deberá contar con un control financiero sistemático que permita tomar decisiones en cuanto a la operatividad de la empresa y evaluar la rentabilidad de la misma.

Sin lugar a dudas una gestión de costos eficaz, contribuye al desarrollo de ventajas competitivas, y promueve una cultura de orden y transparencia. Desde una perspectiva estratégica se aprecia claramente que dicha gestión tiene una marcada influencia en temas de sostenibilidad e impacto organizacional, y esta se evidencia de manera esencial a través de la calidad y oportunidad de la información financiera disponible para apoyar el diseño de estrategias y la adopción de decisiones. 
En el marco de las PYME's artesanales, gran parte no maneja debidamente un control en los costos incurridos de la actividad productiva, y si lo hacen es a nivel empírico.

Briones (citado por Álvarez, W., Baca, L., \& Hernández, G. 2012), alega que el origen familiar de las micro, pequeñas y medianas empresas es muchas veces la principal causa de que estas no lleven un registro contable formal y, para solventar la situación, desde el 2010 el INPYME ${ }^{5}$ en coordinación con el Instituto Nacional Tecnológico (INATEC) desarrollan capacitaciones en tres ejes, que son empresarial, mercadeo y procesos de producción. Evidentemente para que los bancos puedan otorgarles créditos, en su plan de mejora tienen que incluir el componente de la organización de la contabilidad y el INPYME estaría dando seguimiento para que funcionen adecuadamente.

Por tanto, una PYME artesanal tendría un mejor alcance si se aplicara un adecuado sistema de costo, que consagre costos de fábrica, costos de administración, costos de comercialización y costos financieros, esto en conformidad a las políticas de costos.

\section{Caso de aplicación de la estructura de costos de una PYME artesanal dedicada al área de bordado en Masaya}

En el reparto Santa Teresa II etapa del municipio de Masaya, departamento de Masaya, se localiza un pequeño taller manufacturero dedicado al área de bordado artesanal administrado por la propietaria, Señora Bertha Murillo. Fue puesto en marcha con tan solo setecientos córdobas, diez yardas de tela, una caja de seda y una máquina de coser.

La idea emprendedora fue ampliada con un financiamiento de dos mil córdobas obteniendo más responsabilidades y en relación a ello enseño a sus hijas la técnica de bordado para recibir un mayor apoyo en la elaboración de los productos. Actualmente, cuenta con un mejor financiamiento, suficiente maquinaria para el proceso productivo y los servicios de 10 mujeres, de las cuales 6 de ellas cumplen sus labores desde sus propios hogares. La propietaria consta con 10 estilos tanto para niña como para niño entre los 0 y 6 años de edad y es proveedora de 15 tramos en el "Mercado Ernesto Fernández" del mismo departamento.

En cuanto al proceso productivo, se considera igual para todas las prendas, y como muestra se presenta el flujograma de las fases necesarias para obtener el producto terminado.

Cuadro $N^{\circ}$ 1. Flujograma del Proceso Productivo.

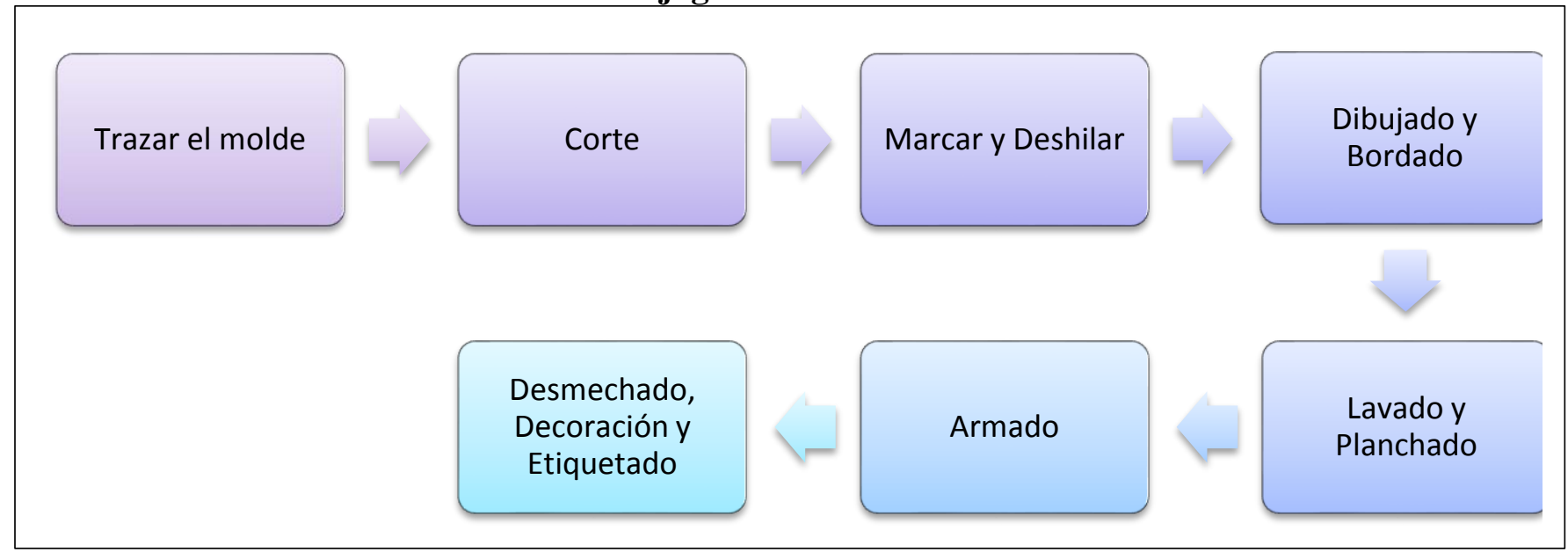


Fuente: Datos proporcionados por la propietaria

Cuadro No. 2. Costos de producción para la elaboración de una cotona

\begin{tabular}{|c|c|c|c|c|}
\hline \multicolumn{5}{|c|}{$\begin{array}{l}\text { HOJA DE COSTOS } \\
\text { Producto: Cotona }\end{array}$} \\
\hline DETALLE & $\mathbf{U} / \mathbf{M}$ & CANTIDAD & $\mathbf{P} / \mathbf{U}$ & $\begin{array}{l}\text { COSTO } \\
\text { TOTAL } \\
\text { C\$ }\end{array}$ \\
\hline \multicolumn{5}{|l|}{ MATERIAL DIRECTO } \\
\hline Manta Hindú & Yarda & 1 & 37 & 37.00 \\
\hline Sub-Total & & & & C\$ 37.00 \\
\hline \multicolumn{5}{|l|}{ MANO DE OBRA DIRECTA } \\
\hline Corte & $\mathrm{H} / \mathrm{h}$ & 1 & 3 & 3 \\
\hline Dibujado & $\mathrm{H} / \mathrm{h}$ & 1 & 0.25 & 0.25 \\
\hline Bordado & $\mathrm{H} / \mathrm{h}$ & 1 & 7 & 7 \\
\hline Armado & $\mathrm{H} / \mathrm{h}$ & 1 & 12 & 12 \\
\hline Decoración & $\mathrm{H} / \mathrm{h}$ & 1 & 1 & 1 \\
\hline Sub-Total & & & & C\$ 23.25 \\
\hline \multicolumn{5}{|l|}{$\begin{array}{l}\text { COSTOS INDIRECTOS DE } \\
\text { FABRICACION }\end{array}$} \\
\hline \multicolumn{5}{|l|}{ MATERIAL INDIRECTO } \\
\hline Seda & Tubo & 0.5 & 10 & 5 \\
\hline Cola de Ratón & Rollo & 0.008 & 65 & 0.5 \\
\hline Chaquiras & Bolsa & 0.002 & 44 & 0.1 \\
\hline
\end{tabular}




\begin{tabular}{|c|c|c|c|c|}
\hline Hilo & Tubo & 0.12 & 8.33 & 1 \\
\hline Elástico & Tubo & 0.01 & 130 & 1.25 \\
\hline Entre tela & Yarda & 0.005 & 22 & 0.1 \\
\hline Sub-total material indirecto & & & & 7.95 \\
\hline \multicolumn{5}{|l|}{ GASTOS OPERATIVOS } \\
\hline Agua & & & & 0.1 \\
\hline Luz & & & & 0.25 \\
\hline Transporte & & & & 0.07 \\
\hline Mantenimiento & & & & 0.15 \\
\hline Depreciación & & & & 0.25 \\
\hline Sub-total gastos operativos & & & & 0.82 \\
\hline Sub-Total & & & & C\$ 8.77 \\
\hline Costo Total & & & & C\$ 69.02 \\
\hline Precio de Venta & & & & C\$ 80.00 \\
\hline Margen de Ganancia & & & & C\$ 10.98 \\
\hline Porcentaje de Ganancia & & & & $16 \%$ \\
\hline
\end{tabular}

Fuente: Datos proporcionados por la propietaria

En complemento de una adecuada estructura de costo es pertinente determinar el costo variable unitario (CVU) para todo proceso productivo, donde se identifican las materias primas que intervienen en el proceso de forma directa y la mano de obra de las trabajadoras que cobran por producción. Además, se deberán determinar los Costos Indirectos de Fabricación, desglosados en Variables y Fijos

Costo Variable Total $=768 * \mathrm{C} \$ 60.25=\mathbf{C} \$$ 46,272.00
Aproximadamente $768 \quad$ piezas mensuales. CVU es equivalente $\mathrm{C} \$ 37.00$ de MD y C\$23.25 de MOD 


\begin{tabular}{|l|l|}
\hline CIF Variables $=\mathrm{C} \$ 7.95 * 768=\mathbf{C} \$ \mathbf{6 , 1 0 5 . 6 0}$ & $\begin{array}{l}\text { para un total de } \mathrm{C} \$ 60.25 . \\
\text { indirectos aplicados a la unidad } \\
\text { producida. }\end{array}$ \\
\hline CIF Fijos $=\mathrm{C} \$ 0.82 * 768=\mathbf{C} \$ \mathbf{6 2 9 . 7 6}$ & $\begin{array}{l}\text { Se obtuvo } \mathrm{C} \$ 0.82 \text { de CIF Fijos por } \\
\text { pieza }\end{array}$ \\
\hline CIF Totales $=\mathrm{C} \$ 8.77 * 768=\mathbf{C} \$ \mathbf{6 , 7 3 5 . 3 6}$ & $\begin{array}{l}\mathrm{C} \$ 7.95+\mathrm{C} \$ 0.82 \text { da resultado de los } \\
\mathrm{CIF} \text { Totales. }\end{array}$ \\
\hline Costo Total Unitario $=\mathrm{C} \$ 60.25+\mathrm{C} \$ 8.77=\mathbf{C} \$ \mathbf{6 9 . 0 2}$ \\
\hline
\end{tabular}

Determinar el margen de contribución que conlleva la elaboración del producto, permite evaluar el nivel de rentabilidad de dicha actividad manufacturera. El resultado de este concepto se obtiene restando al precio de venta unitario el costo variable unitario, integrado por los costos directos. Cabe señalar que, el margen de contribución permite cubrir el costo fijo que implico elaborar la pieza

\section{Margen de Contribución $=\mathbf{C} \$ 80-\mathrm{C} \$ 60.25=\mathrm{C} \$ 19.75$}

En seguimiento a los datos anteriores es pertinente identificar el punto de equilibrio para la elaboración de este producto, y la relevancia de este dato recae hasta el punto de establecer el mínimo de unidades a producir sin obtener pérdida ni ganancia. Para este caso sería el siguiente:

\section{Punto de equilibrio= C $\$ 629.76$ (Costo Fijo Total) = $31.89 \sim 32$ unidades. \\ C\$19.75 (Margen de Contribución)}


Cuadro No. 3. Resultados generados en relación a una producción estimada ensual

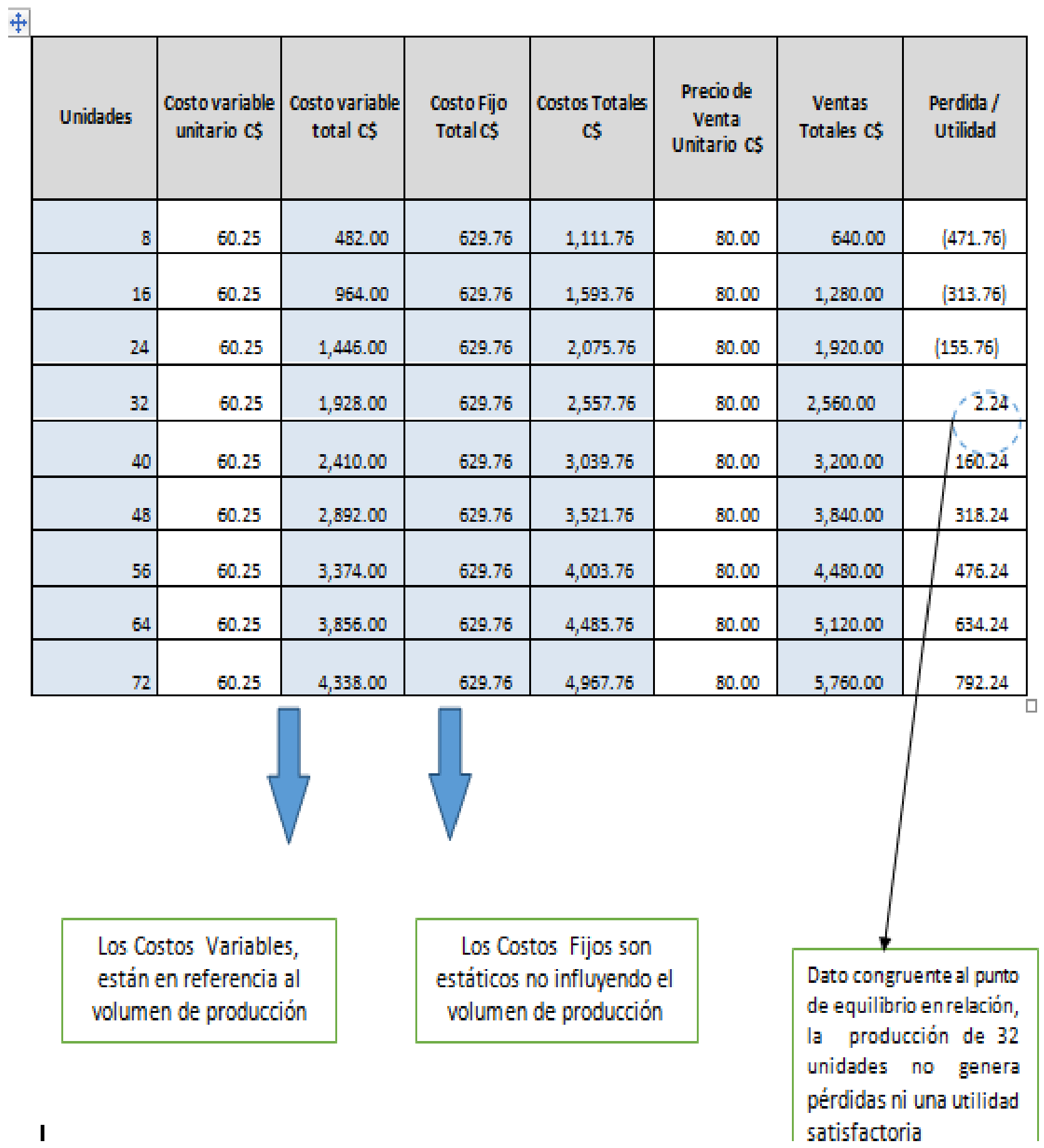


Entonces en relación datos proporcionados con anterioridad podemos obtener los costos totales y la utilidad generada basándonos en una producción estimada de 768 unidades mensuales, y en síntesis tendríamos lo siguiente:

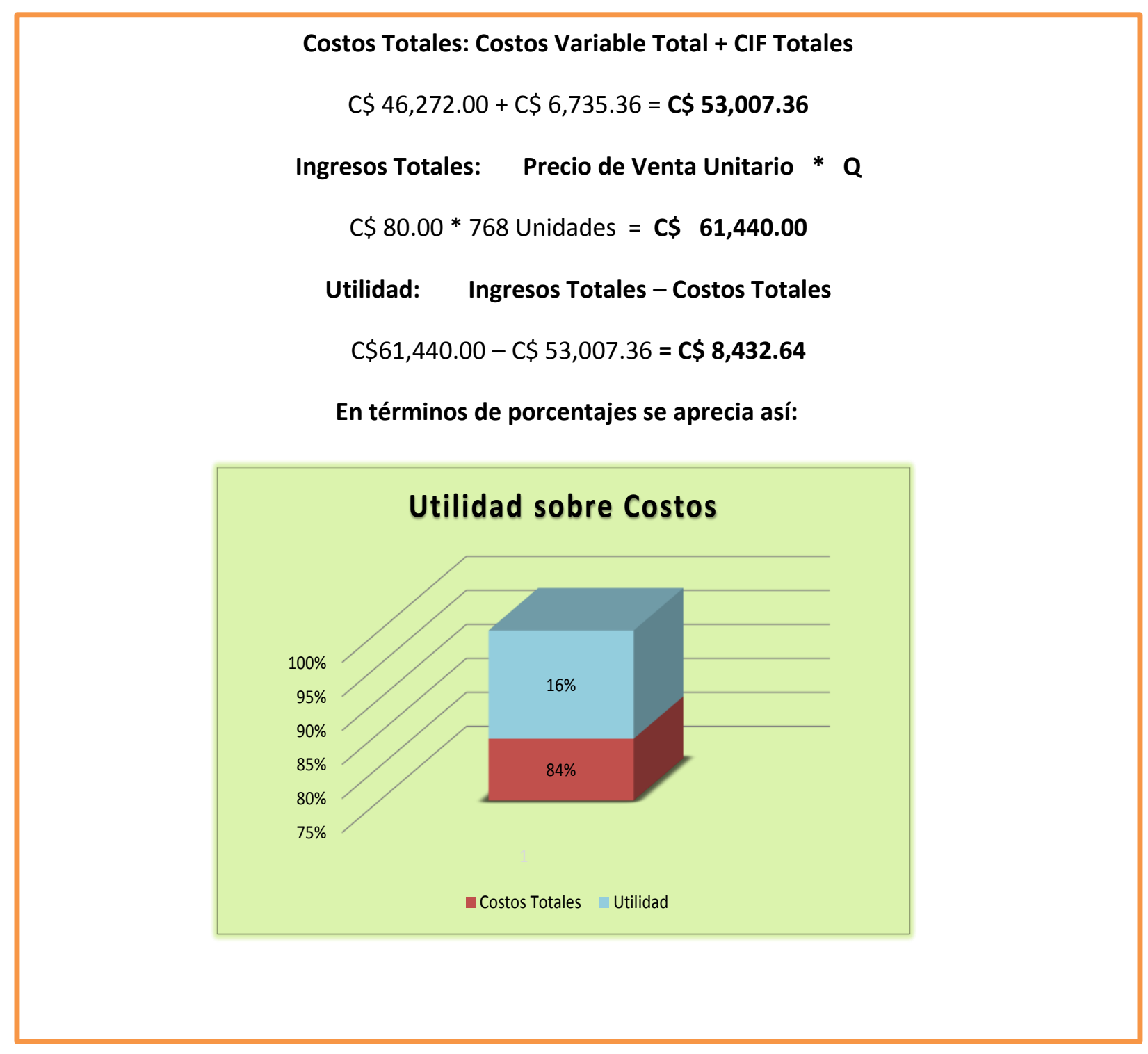

Fuente: Elaboración Propia

\section{CONCLUSIONES}

Las PYME's artesanales del departamento de Masaya carecen de conocimientos solidificados en relación al control financiero, técnicas de comercialización y escaso apoyo financiero para la 
continuidad de sus actividades artesanales. El caso de aplicación no fue la excepción, puesto que, se identificó la falta de un adecuado manejo financiero, siendo este el instrumento esencial como parte de la planificación operativa y para ejecución tanto interna como externa.

Por consiguiente, aunque el aporte económico de la actividad artesanal sea considerable en relación a la economía nacional, la situación es discrepante debido a que la capacitación técnica para este sector no es ampliado, pues únicamente la producción se estanca en un simple abastecimiento económico y más cuando la mayoría de estas PYME’s son de carácter familiar.

\section{REFERENCIAS}

Lacayo, L. N. (2013, 3 de mayo). En Nicaragua existen 121,919 empresas. El nuevo diario. Recuperado de http://www.elnuevodiario.com.ni/economia/284929

La voz del Sandinismo. (2012). Crecen pequeñas empresas de Nicaragua en un 6\% en este 2012. Recuperado de http://www.lavozdelsandinismo.com/economia/2012-11-10/crecen-pequenasempresas-de-nicaragua-en-un-6-en-este-2012/

Álvarez, W., Baca, L., \& Hernández, G. (2012, 3 de mayo).Mayoría de empresas sin contabilidad. La prensa. Recuperado dehttp://www.lavozdelsandinismo.com/economia/2012-1110/crecen-pequenas-empresas-de-nicaragua-en-un-6-en-este-2012/

Casa de gobierno. (2012). Nuevo Ministerio en fomento a la Economía Familiar, Comunitaria,

Cooperativa y Asociativa. Managua. Autor. Recuperado de
http://www.tortillaconsal.com/nicaragua_triunfa_72.doc 